\title{
Factores de riesgo de la caries de la infancia temprana relacionados a hábitos de crianza en Latinoamérica
}

\author{
Risk factors for early childhood caries \\ related parenting habits in Latin America \\ Dhttps://orcid.org/0000-0002-1484-9852 \\ https://orcid.org/0000-0001-6493-4687 \\ https://orcid.org/0000-0003-4281-724
}

Sofía Ballesteros Ramírez ${ }^{1 a}$

Salomé Manzano Saldarriaga ${ }^{1 a}$

Gloria Emilsen Pabón ${ }^{1 \mathrm{bc}}$

Correspondencia: sballesteros12@estudiantes.areandina.edu.co

\section{Resumen}

Objetivo: Determinar los factores de riesgo causantes de la caries de la infancia temprana (CIT) en niños, entre el nacimiento y los 5 años de edad, de Latinoamérica, en los últimos 10 años. Metodología: Se realizó un estudio cuantitativo descriptivo tipo scoping, donde se llevó a cabo una búsqueda en Science Direct, PubMed, SciELO, Google Scholar, Proquest, desde el 2011 hasta el 2021, incluyendo artículos en inglés, portugués y español. Resultados: En 20 artículos se evaluaron los conocimientos y prácticas de los padres y/o cuidadores, donde se analizó si tenían o no buenos hábitos de higiene bucal, siendo este uno de los factores de riesgo más predominante. Conclusión: Se evidencia que entre los principales riesgos de la CIT se tiene una dieta rica en carbohidratos, permisividad con el lactante del consumo de bebidas azucaradas por las noches, utilizar el biberón después de un año de vida, presentar una mala higiene oral y el factor socioeconómico, que resultó el más predisponente. Finalmente, se pudo determinar que existe una relación entre los hábitos de crianza y la CIT.

Palabras clave: caries dental, factores de riesgo, hábitos, preescolar

\section{Abstract}

Objective: To determine the risk factors that cause early childhood caries (ECC) in children, between birth and 5 years of age, in Latin America, in the last 10 years. Methodology: A quantitative descriptive scope study was carried out, where a search was conducted in Science Direct, PubMed, SciELO, Google Scholar, Proquest, from 2011 to 2021, including articles in English, Portuguese and Spanish. Results: In 20 articles, the knowledge and practices of parents and/or caregivers were evaluated, where it was analyzed whether or not they had good oral hygiene habits, this being one of the most predominant risk factors. Conclusion: It is evident that among the main risks of ECC are a diet rich in carbohydrates, permissiveness with the infant of the consumption of sugary drinks at night, using the bottle after one year of life, presenting poor oral hygiene and the socioeconomic factor, which was the most predisposing. Finally, it was possible to determine that there is a relationship between parenting habits and ECC.

Keywords: dental caries, risk factors, habits, preschool

\footnotetext{
${ }^{1}$ Fundación Universitaria del Área Andina. Facultad de Odontología. Pereira, Colombia

a Estudiante

${ }^{b}$ Docente de la Facultad de Odontología. Tutora encargada del artículo

c Rehabilitadora oral
} 


\section{Introducción}

En el presente artículo se pretende identificar los factores de riesgo de la caries en la infancia temprana (CIT, por sus siglas en español) relacionados con los hábitos de crianza; por lo que se requiere del manejo de una serie de conceptos, como el de la caries en tan temprana edad; por lo que "la Academia Americana de Odontología Pediátrica define la caries de la infancia temprana como la presencia de uno o más dientes cariados (cavitados o no), ausentes (debido a caries) o restaurados en la dentición primaria", ${ }^{1}$ en niños de edad preescolar, esto es, entre el nacimiento y los 5 años de edad.

De igual manera, y en razón de que la caries dental se considera como un problema de salud pública en países subdesarrollados, como lo manifiestan Aguilar Ayala et al., ${ }^{2}$ esta actúa sobre el progreso y la calidad de vida de los niños que la padecen, siendo una enfermedad crónica, pero prevenible.

A lo cual, Macías et al. ${ }^{3}$ refieren que la progresión o evolución de estas lesiones (CIT) traen consecuencias locales y sistémicas a corto, mediano y largo plazo; siendo algunas de las complicaciones locales la infección, inflamación, pérdida de espacio por exodoncias prematuras, alteraciones en la masticación, hasta deficiencias en el desarrollo del habla del menor; asimismo, las consecuencias sistémicas pueden incluir problemas de aprendizaje, ansiedad, agresividad, déficit de atención, hiperactividad, alteraciones en el sueño y hasta desórdenes gastrointestinales por un hábito inadecuado de alimentación.

Por consiguiente, al avanzar la caries de la infancia temprana, los niños pueden presentar complicaciones a largo plazo, llegando a afectar su salud general. Esta lesión tiene mayor relevancia en las poblaciones más vulnerables, siendo un factor de riesgo el tener limitado el acceso a una adecuada atención en los servicios de salud.

Por otro lado, Parra et al. ${ }^{4}$ afirman que América Latina es la región que presenta un porcentaje de 44 al $93 \%$ de prevalencia de la caries de la infancia temprana, donde los factores de riesgo son agentes causales para desencadenarla, entre los que se tienen como factores predominantes una inadecuada higiene oral, la presencia de placa y lo más preocupante, el desconocimiento de los padres y/o cuidadores de los infantes.

A lo que se puede añadir que "la razón principal para la prevención y tratamiento de la caries en la dentición primaria es para evitar el dolor y la infección, ya que esto ocasiona un impacto considerable en el bienestar psicosocial de los niños y puede afectar el desempeño de las actividades de la vida diaria". ${ }^{5}$

Por lo tanto, al tener un índice bastante alto de niños afectados por la caries de la infancia temprana en diferentes países latinoamericanos, se asume que se siguen presentando falencias con respecto a la prevención brindada a la comunidad, lo que tiene como consecuencias directas afecciones que dificultan las actividades de esta población en el día a día.

\section{Justificación}

Aguilar Ayala ${ }^{2}$ establece que la caries de la infancia temprana (CIT) es reconocida como un problema de salud pública, por su alta prevalencia y el impacto que tiene respecto a la salud y la calidad de vida de los niños que la desarrollan.

Por tanto, la CIT afecta a gran parte de Latinoamérica, con las consecuentes desventajas para esta población que no cuentan con el apoyo y la información oportuna de programas de prevención. Cabrera Escobar et al. ${ }^{6}$ comentan que este problema de salud pública ha generado gran atención en la literatura científica; mostrando cómo esta enfermedad multifactorial prevalece en diversas poblaciones.

De tal manera, este estudio se realiza con el fin de conocer algunos de los hábitos más sobresalientes que practican los padres y/o cuidadores de los infantes, que pueden tener influencia en el desarrollo de la 
caries de la infancia temprana; teniendo en cuenta que las poblaciones vulnerables pueden presentar más factores de riesgo como el factor socioeconómico, una mala alimentación, malas prácticas de higiene oral y una limitada información con respecto a programas de prevención.

Asimismo, según el informe publicado por la Organización Mundial de la Salud (OMS), "la presencia de enfermedades bucodentales es alta en especial en los grupos de población vulnerable y pobre, tanto en los países en vías de desarrollo como en los desarrollados".7

Finalmente, este artículo resulta de especial significancia en tanto plantea información de calidad para la población que tenga acceso a él, incluyendo, de forma indirecta, una prevención primaria para el entorno que rodea al individuo.

\section{Sistematización del problema}

Los factores de riesgo, según Beraldi $\mathrm{Ml}$ et al., ${ }^{8}$ no, necesariamente, son el origen responsable, sino que se relacionan con el evento.

Para Montero Canseco Delia et al. 9 "Los factores primarios de riesgo (...) incluyen: Alimentar (...) con biberón cuyo contenido incluya algún líquido azucarado durante la siesta o por la noche; permitir que un lactante se alimente del seno materno libremente (free demand) (...); seguir utilizando el biberón después del primer año de vida; falta o ausencia de limpieza dental por parte de los padres y factores socioeconómicos".

Según González, ${ }^{10}$ la cariogenicidad de un alimento no se determina por el contenido de azúcar presente, sino también por las características físicas del alimento, como retención, capacidad para estimular el flujo salival y cambios químicos en la saliva, así como textura, frecuencia, horario de consumo, tiempo de permanencia en la boca y que la sacarosa es la azúcar más cariogénica.

Arango De La Cruz MC et al. ${ }^{11}$ sugieren que para el desarrollo de la caries dental no es importante solo el consumo de azúcar, sino también el tiempo de exposición a los alimentos; relacionándose de manera directa con la caries de la infancia temprana.

Por otra parte, como expresa Gonzalez, ${ }^{10}$ en pediatría la educación del niño y su entorno familiar es uno de los factores más importantes para la prevención y la promoción de la salud. Los niños y sus padres deben comprender sus responsabilidades y necesidades en el mantenimiento de la salud.

La literatura relacionada con el tema da a conocer que existe un alto índice de caries en los menores de 5 años de edad que puede ser ocasionada porque los padres y/o acudientes (entorno familiar) no están recibiendo una adecuada información, por cuanto manifiestan Herney Alonso y Lida Marcos Muñoz ${ }^{12}$ sobre los conocimientos previos, prácticas y variables sociodemográficas de las madres con respecto a la salud bucodental de sus hijos son factores importantes para la salud pública a nivel del país y de la región, teniendo en cuenta la influencia que ellas tienen en temas como la cultura y la salud, siendo las que transmiten comportamientos que llevan a futuros buenos hábitos.

Entre tanto, al no brindarse una adecuada información a los padres de familia, los infantes se ven directamente afectados, desde una mala salud oral, hasta una mala calidad de vida, evidenciándose esta problemática en diferentes países de América Latina. 


\section{Metodología}

Se llevó a cabo un estudio cuantitativo-descriptivo tipo scoping review, donde se realizó una búsqueda en Science Direct, PubMed, SciELO, Google Scholar, Proquest, para el periodo 2011-2021, que incluyó artículos en español, inglés y portugués. De 45 artículos fueron incluidos 30 artículos que cumplen con los criterios de inclusión estipulados y fueron excluidos 15 artículos que estaban fechados antes del 2011; se citaron 30 en total; así mismo, este proyecto fue aprobado por el comité de la Fundación Universitaria del Área Andina-seccional Pereira.

Esta revisión de la literatura se realiza para poder determinar factores de riesgo y causas desencadenantes de la CIT y, a su vez, identificar posibles hábitos de crianza que influyan en el desarrollo de la caries de la infancia temprana.

\section{Aspectos teóricos}

\section{La caries}

La caries dental es considerada como la enfermedad de mayor peso, ya que esta afección puede llegar a afectar las condiciones de la salud general del infante. Al cabo de algunos años se reportó, según los centros de control y prevención de enfermedades, ${ }^{8}$ que la prevalencia de esta caries ha aumentado en los últimos años, siendo la enfermedad más prevalente en los niños menores de 5 años de edad (edades tempranas) en los Estados Unidos.

Al respecto, comentan Loreto Núñez et al. ${ }^{13}$ que la caries es la enfermedad más frecuente a lo largo de la infancia, trayendo consecuencias a los niños, desde una desmineralización del esmalte, hasta la pérdida de algunos o quizá todos sus dientes.

También, existen evidencian de que "en el Perú, la enfermedad que más afecta la salud oral en la población infantil es la caries dental, cuya prevalencia es del $59.1 \%$ en dentición decidua y $85.6 \%$ en dentición mixta, siendo una población con mayor riesgo de tener caries". ${ }^{5}$

\section{Caries de la infancia temprana}

Según Tovar Zevallos, ${ }^{1}$ la etiología de la caries de aparición temprana es multifactorial, por ende, los diferentes factores etiológicos pueden diferir de un niño a otro. No hay un factor de riesgo único con suficiente dominio predictivo para seleccionar a algún lactante o infante en una posición de riesgo.

Con lo anterior, se genera una preocupación, con respecto a la salud oral de los niños, teniendo diversos factores de riesgo a su alrededor; por ejemplo, Pardo Herrera et al., ${ }^{14}$ en 2011, encontraron que los niños que presentaban una mala salud oral tenían tres veces más posibilidad de faltar a la escuela que los menores que tenían una buena salud oral; viéndose afectado el desempeño escolar de los niños que presentan la caries de la infancia temprana.

De otra parte, Ramírez-Puerta BS el al. ${ }^{15}$ mencionan que "la salud bucal durante los primeros años de vida -en su estrecha relación con la salud general- ha sido motivo de preocupación en muchos países, donde la caries dental continúa siendo la enfermedad crónica más común y el principal problema de salud bucal de la infancia". 


\section{Hábitos y caries de la infancia temprana}

Los hábitos y la caries de la infancia temprana están directamente relacionados; de tal manera que, según Levin $A,{ }^{16}$ el rol de los padres y los factores familiares como los psicosociales, el funcionamiento familiar, los hábitos odontológicos y el estrés de estos en la crianza de sus hijos son relevantes en la experiencia de caries de los niños y condicionarán un aumento en la probabilidad de presentar CIT, por lo que es posible que aumente el factor de riesgo que rodea al infante.

Algunos hábitos que se perciben como buenos son posibles factores de riesgo que desencadenan la caries de la infancia temprana; por lo que, ante esta problemática, se intenta identificar qué puede desarrollar más fácil estas lesiones para dar información clara y concisa a las poblaciones, ya que "las políticas y programas de salud futuros deben estar dirigidos a los niveles de la familia y la comunidad". ${ }^{16}$

Se puede identificar que la influencia que tienen los padres y/o cuidadores de los niños cumple un rol importante; teniendo en cuenta que se realicen buenos hábitos, se podría disminuir el índice de caries de la infancia temprana; trayendo beneficios a su calidad de vida.

Además de una escasa información se presentan otros factores que pueden afectar negativamente en la salud oral del menor, como lo son los factores psicosociales; entre estos se "encuentran la depresión materna, bajo sentido de coherencia, habilidades internas y externas de las personas para manejar las tensiones, paternidad indulgente y estrés parental". ${ }^{17}$

\section{Resultados}

Se obtuvo un total de 45 artículos, donde 30 artículos obtenían los requisitos de inclusión, los cuales fueron incluidos en esta revisión y 15 artículos fueron excluidos, ya que no cumplían con ciertos criterios necesarios.

Teniendo en cuenta los resultados derivados de la revisión de artículos se halló lo siguiente:

- En 6 artículos evaluados por medio de exámenes clínicos intraorales y que fueron acompañados de encuestas y cuestionarios se concluyó que existe un alto índice de prevalencia determinando como principales factores de riesgo: alimentar al niño con biberón, cuyo contenido incluya algún líquido azucarado, durante la siesta o por la noche, permitir que el lactante se alimente del seno materno libremente durante la noche, seguir utilizando el biberón después del primer año de vida, falta o ausencia de limpieza dental por parte de los padres y/o acudientes, una educación limitada materna, una dieta alta en carbohidratos, falta de calcio o micronutrientes y factores socioeconómicos o socioculturales.

- Cuatro artículos analizaron y describieron las causas más frecuentes que desencadenan la caries de la infancia temprana en los menores de 5 años, como una dieta alta en carbohidratos, una deficiente higiene oral, el uso extendido del biberón; también, su etiología se puede describir por medio de tres factores importantes como lo son: el huésped (teniendo una superficie dentaria susceptible), la higiene oral (siendo deficiente) y el consumo frecuente y alto de carbohidratos.

- Se confrontan diferentes etiologías, de las cuales se desprenden otras causas, más factores que pueden desencadenar la caries de la infancia temprana, como lo son las bacterias que afectan la boca del niño y la técnica de cepillado; ante lo cual se concluye que se deben promocionar estrategias de prevención, para así lograr conseguir una buena higiene oral en los niños de infancia temprana a tiempo.

- Por último, en 20 artículos se evaluaron conocimientos y prácticas de los padres y/o cuidadores por medio de cuestionarios, entrevistas y exámenes intraorales a los infantes, donde se analizó si los padres de familia tenían buenos hábitos de higiene oral, para saber si los infantes se encuentran 
expuestos a la caries de la infancia temprana; de tal manera que se evidenció que la caries de la infancia temprana tiene relación con los hábitos de crianza del hogar, concluyendo que algunos padres tienen buen conocimiento sobre la enfermedad, donde la minoría de padres o madres llevan a los niños a servicios de prevención; también se evidenció que las prácticas y hábitos de algunos padres tienen un aspecto negativo para el infante; por lo cual se necesita de la promoción e implementación de políticas nutricionales más estrictas para reducir el consumo de azúcar de los niños menores de 5 años.

\section{Figura 1}

Porcentajes finales

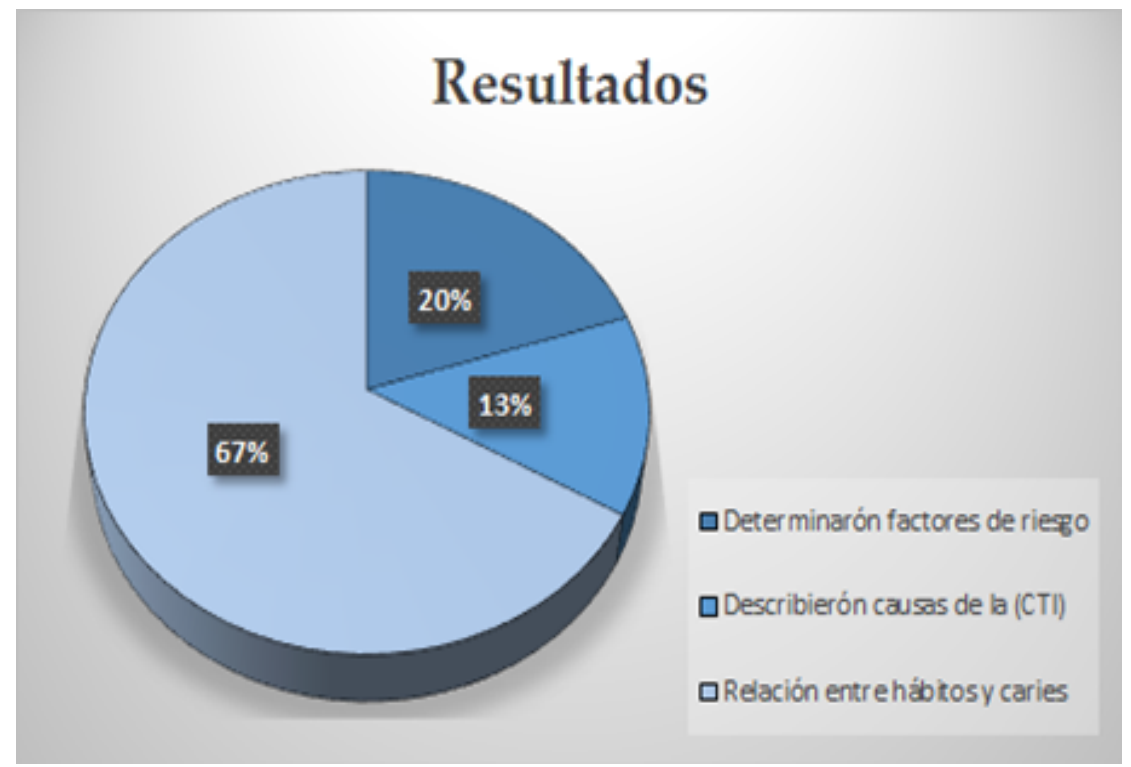

Discusión

Martínez et al., en el año 2011, en su estudio denominado Conocimientos, actitudes y prácticas en salud bucal de padres y cuidadores en hogares infantiles, Colombia, ${ }^{18}$ buscaban relatar condiciones y experiencias en salud oral de padres y/o cuidadores, a través de entrevistas y encuestas. Luego del análisis se observó que los padres tenían buenos horizontes de instrucción $(58.9 \%)$ y cualidades propicias $(74.5 \%)$. Concluyendo así que los papás son los factores primordiales a la hora de efectuar prácticas orales saludables y fomentar los buenos hábitos del menor a futuro.

Por su parte, Aguilar Ayala et al., ${ }^{2}$ en el año 2014, analizaron la prevalencia de caries y los factores de riesgo en pacientes menores de 48 meses llevando a cabo exámenes clínicos de los menores y cuestionarios para los padres de familia, y concluyeron que, debido a un alto índice de prevalencia de mancha blanca, se deben implementar más programas preventivos, donde el autocuidado juega un papel importante, como estrategia de prevención.

Por otro lado, Tovar Zevallos et al., en el año 2015, en su estudio Prevalencia de caries de aparición temprana en niños de 3 a 5 años y 11 meses de edad y su asociación con sus factores predisponentes en el policlínico EsSalud Chincha de septiembre 2012 a febrero $2013,{ }^{1}$ buscaba establecer la prevalencia de caries de aparición temprana (CAT) en niños de 3 a 5 años en Perú; donde se evaluaron variables como la edad, el género, índice de mala higiene oral, frecuencia de bebidas azucaradas, presentando prevalencia de caries de $67.33 \%$, presentando un valor elevado para edades tempranas. 
Finalmente, en el año 2020 se realiza un estudio muy importante en Perú, titulado Calidad de vida relacionada a la salud bucal en niños peruanos con caries de la infancia temprana severa, ${ }^{5}$ en el que se pretendió evaluar la percepción parenteral sobre la calidad de vida, relacionada a la salud bucal, en niños de 3 a 5 años sin y con caries de la infancia temprana, donde se aplicó un instrumento que fue el ECOHIS; con esta investigación concluyeron que la relación que tiene la calidad de vida con la salud bucal fue negativamente afectada por la CIT.

Asimismo, los estudios concuerdan en que la caries dental se deduce como la enfermedad de magno peso, en relación a la salud bucal, en Latinoamérica.

\section{Conclusión}

Con lo planteado anteriormente, se puede concluir que los principales factores de riesgo de los niños menores de 5 años fueron: una dieta rica en carbohidratos, permitir que el lactante consuma bebidas azucaradas en las noches, utilizar el biberón por más de un año, presentar una mala higiene oral y el factor socioeconómico, siendo este último el más predisponente.

Por otra parte, un defecto en el esmalte dental, prácticas inadecuadas de higiene oral y el bajo peso al nacer se asocian a las causas más frecuentes de la caries de la infancia temprana, presentando como consecuencia un alto porcentaje de prevalencia de esta enfermedad; teniendo predilección por las poblaciones más vulnerables y pobres en Latinoamérica.

Finalmente, se pudo identificar que existe una relación entre los hábitos de crianza y la caries de la infancia temprana, donde estos hábitos de crianza afectan negativamente la salud oral de los infantes.

\section{Referencias}

1. Tovar Zevallos MA. Prevalencia de caries de aparición temprana en niños de 3 a 5 años y 11 meses de edad y su asociación con sus factores predisponentes en el policlínico Essalud Chincha de septiembre 2012 a febrero 2013. Univ Peru Ciencias Apl [Internet]. 2015 [cited 2021 Mar 20]; Available from: https:// repositorioacademico.upc.edu.pe/handle/10757/581

2. Aguilar-Ayala, Fernando Javier, Duarte-Escobedo, Claudia Gabriela, Rejón-Peraza, Marina Eduviges, Serrano-Piña, Rodrigo, \& Pinzón-Te, Alicia Leonor. (2014). Prevalencia de caries de la infancia temprana y factores de riesgo asociados. Acta pediátrica de México, 35(4), 259-266. Recuperado en 22 de febrero de 2021.

3. Macías C, Díaz D, Caycedo M, Lamus F, Rincón C. Asociación De Caries De Infancia Temprana Con Factores De Riesgo En Hogares Comunitarios Del Instituto Colombiano De Bienestar Familiar En Zipaquirá, Colombia/Association Of Early Childhood Caries With Risk Factors At Community Homes Of Instituto Colombiano De Bienestar Familiar In Zipaquirá, Colombia. Revista de la Facultad de Odontología Universidad de Antioquia 2016; 28 (1):123-138.

4. Parra-Coronel J, Astudillo-Neira D, Ortiz-Ulloa J. Early childhood caries and risk factors in young children of medium-low socioeconomic status, Ecuador. Revista Investigación en Salud Universidad de Boyacá [Internet]. 2020 Mar 25 [cited 2021 Sep 3];7(1):52-70. Available from: https://revistasdigitales.uniboyaca. edu.co/index.php/rs/article/view/451/53

5. Munayco-Pantoja Evelyn R., Pereyra-Zaldívar Héctor, Cadillo-Ibarra Marleny M. Calidad de vida relacionada a la salud bucal en niños peruanos con caries de infancia temprana severa. Odontoestomatología [Internet]. 2020 [citado 2021 Feb 22]; 22 (36): 4-14. Disponible en: http://www.scielo.edu.uy/scielo. php?script=sci_arttext\&pid=S1688-93392020000300004\&lng=es. Epub 01-Dic-2020. http://dx.doi. org/10.22592/ode2020n36a2

6. Cabrera Escobar Deyanira, López García Félix, Ferrer Hurtado Orquídea, Tellería Castellanos Ana María, Calá Domínguez Tony. Factores de riesgo de caries dental en niños de la infancia temprana. Paulo VI. Venezuela. 2012. Rev.Med.Electrón. [Internet]. 2018 Ago [citado 2021 Mar 16]; 40(4): 958-967. Disponible 
en :http://scielo.sld.cu/scielo.php?script=sci_arttext\&pid=S1684-18242018000400004\&lng=es

7. Herrera I. Ingresar [Internet]. Www-sciencedirect-com.proxy.bidig.areandina.edu.co. 2021 [cited 13 March 2021]. Available from: https://www-sciencedirect-com.proxy.bidig.areandina.edu.co/science/article/pii/ S1665706317300192

8. Beraldi MI, Shultz Martins Pio M, Dalledone Codascki M, Guerrart Portugal ME, Vida Cassi Bettega P. Cárie na primeira infância: uma revisão de literatura. Revista Gestão e Saúde [Internet]. 2020 [cited 2021 May 2];2(22). Available from: http://www.herrero.com.br/files/revista/file07edddf2dfc55d012612cbbcb7e78fc7. pdf

9. Montero Canseco Delia, López Morales Patricia, Castrejón Pérez Roberto Carlos. Prevalencia de caries de la infancia temprana y nivel socioeconómico familiar. Rev. Odont. Mex [revista en la Internet]. 2011 Jun [citado $2021 \mathrm{Feb} 22$ ]; 15 ( 2 ): 96-102.

10. González Castro GE. Jardín Infantil de la Universidad Nacional de Colombia: evaluación de un programa para la promoción de salud bucal en la primera infancia / Kindergarten of the National University of Colombia: Evaluation of a Health Promotion Program for the Early [...]. Universitas Odontológica 2012;31(66).

11. Arango De La Cruz MC, Jaramillo Echeverry A, Cruz Valderrama CA. Prevalencia de Caries de la Infancia Temprana y Factores de Riesgo Asociados en Niños de 1 a 5 Años en la Ciudad de Santiago de Cali. Ustasalud. 2013;12(2):108

12. Herney Alonso RR, Lida Margot Muñoz Ordóñez. Creencias, conocimientos y prácticas de madres respecto a la salud bucal en Popayán, Colombia. Universitas Odontologica 2019;38(80).

13. Loreto Núñez F, Javier Sanz B, Gloria Mejía L. Caries dental y desarrollo infantil temprano estudio piloto. Rev Chil Pediatr. 2015.

14. Pardo Herrera I, Cortes Cáceres A, Mosquera Mena AC, Vergara Zarate A. Factores de riesgo para caries en niños que asistieron a la clínica odontológica de la universidad santiago de cali. Arch venez farmacol ter [Internet]. 2019 [citado el 21 de octubre de 2021]; Disponible en: https://repository.usc.edu. co/handle/20.500.12421/2666

15. Ramírez-Puerta BS, Franco-Cortés AM, Ochoa-Acosta E, Escobar-Paucar G. Experiencia de caries en dentición primaria en niños de 5 años, Medellín, Colombia */Caries experience in primary dentition among 5-year-olds in Medellin, Colombia/Presença de cáries em dentição primária em crianças de 5 anos, Medellín, Colômbia. Revista de la Facultad Nacional de Salud Pública 2015 Sep;33(3):345-352.

16. Levin A, Sokal-Gutierrez K, Hargrave A, Funsch E, Hoeft KS. Maintaining traditions: A qualitative study of early childhood caries risk and protective factors in an indigenous community. Int J Environ Res Public Health [Internet]. 2017;14(8). Disponible en: http://dx.doi.org/10.3390/ijerph14080907.

17. Hofer-Durán Paulina, Nuñez Javiera, Atala-Acevedo Claudia, Espinoza-Espinoza Gerardo, SinsaySchmeisser Javiera, Zaror Carlos. Ausencia de Asociación entre Estrés Parental y Caries Temprana de la Infancia en Preescolares. Int. J. Odontostomat. [Internet]. 2020 Jun [citado 2021 Mar 23]; 14 (2): 220-229.

18. Martínez FG, Barrios CCS, Salinas LEM. Conocimientos, actitudes y prácticas en salud bucal de padres y cuidadores en hogares infantiles, Colombia. Salud Pública de México [Internet]. 2011 Jun 1 [cited 2021 Sep 8];53:247-57. Available from: https://scielosp.org/article/spm/2011.v53n3/247-257/

- Conflicto de intereses: La presente investigación no presenta conflicto de intereses entre los investigadores.

- Fuente de financiamiento: La presente investigación fue financiada por los investigadores. 